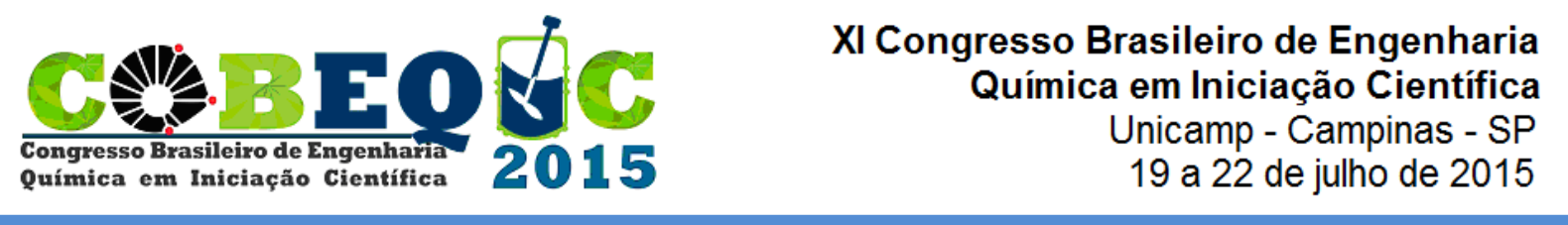

\title{
ESTUDO DO DESEMPENHO DO ROTATING BIOLOGICAL CONTACT (RBC) NA REMOÇÃO DE CARGA ORGÂNICA DE EFLUENTES LÍQUIDOS HOSPITALARES
}

\author{
N. dos SANTOS ${ }^{1}$, F.L. CARVALHO ${ }^{2}$, D.J. CATELAN ${ }^{3}$, B.V. da ROSA ${ }^{4}$, D.D. da \\ SILVEIRA $^{5}$

\begin{abstract}
${ }^{1}$ UFSM/Engenharia Química - E-mail : nortondossantos@hotmail.com ${ }^{2}$ UFSM/Engenharia Química - E-mail : fernando.carvalho.198@gmail.com

${ }_{3}^{3}$ UFSM/Engenharia Química - E-mail :douglas.jauris@hotmail.com

${ }^{4}$ UFSM/Engenharia Química - E-mail : brbvgs@hotmail.com

${ }^{5}$ UFSM/Dept. Engenharia Química - E-mail : djalma.silveira@terra.com.br
\end{abstract}

\begin{abstract}
RESUMO - A escassez de recursos hídricos e a crise enérgica vêm gerando uma busca por tecnologias para o tratamento de efluentes urbanos e industriais que tenham bom desempenho na degradação de matéria orgânica e também tenham baixo consumo energético. Incluindo-se neste contexto atual, o presente trabalho objetiva a avaliação da eficiência de um Reator Biológico de Contato (RBC) para o tratamento do efluente gerado no Hospital Universitário de Santa Maria (HUSM). O RBC consiste em um tratamento biológico aeróbio contendo um conjunto de discos rotatórios (biodiscos) como suporte para os microrganismos formando um biofilme. A degradação da matéria orgânica ocorre no biofilme parcialmente submerso no substrato. O RBC alvo deste estudo foi construído em escala de bancada, portando 30 discos, com uma área de superfície total de aproximadamente $0,68 \mathrm{~m}^{2}$, um volume útil de 5 litros e operando a uma vazão de 1 litro por hora. Os parâmetros analisados, conforme o Standard Methods for Examination of Water and Wastewater, foram a $\mathrm{DQO}$ e a $\mathrm{DBO}_{5}$. As eficiências médias de degradação da $\mathrm{DQO}$ e da $\mathrm{DBO}_{5}$ foram respectivamente: $77 \%$ e $89,6 \%$. A partir das boas eficiências de degradação da $\mathrm{DQO}$ e da $\mathrm{DBO}_{5}$, conclui-se que os Reatores de Biológicos de Contato são uma boa opção para o tratamento de efluentes hospitalares.
\end{abstract}

\section{INTRODUÇÃO}

A preocupação com a qualidade da água e dos seus mananciais vem ganhando atenção no decorrer dos últimos anos. Devido a falta de tratamento dos efluentes gerados por diversas fontes o meio em que vivemos acaba recebendo essa carga poluidora e pondo e em risco a saúde de todos aqueles que o habitam.

Assim a contaminação causada pelos hospitais tem tido uma preocupação crescente, tanto no Brasil como na Europa e América do Norte (Kümmerer, 2004). Uma vez que a carga 


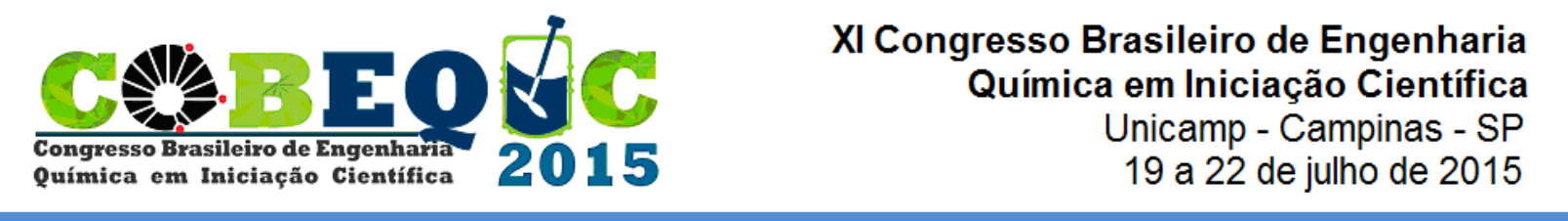

poluidora oriunda não contem apenas matéria orgânica e nitrogenada, mas também resquícios de medicamentos o que torna indispensável o tratamento do efluente hospitalar.

Segundo MINETTO, L. (2009) a ordem de grandeza de alguns parâmetros do efluente gerado pelo Hospital Universitário de Santa Maria pode ser visto na tabela 1.

Tabela 01- Caracterização de efluente hospitalar (HUSM)

\begin{tabular}{|c|c|}
\hline Parâmetro & Valor \\
\hline $\mathrm{DBO}_{5}\left(\mathrm{mg} \mathrm{O}_{2} \mathrm{~L}^{-1}\right)$ & 303,7 \\
\hline $\mathrm{DQO}\left(\mathrm{mg} \mathrm{L}^{-1}\right)$ & 420.0 \\
\hline Matéria Orgânica $\left(\mathrm{mg} \mathrm{L}^{-1}\right)$ & 130,5 \\
\hline Nitrogênio Amoniacal $\left(\mathrm{mg} \mathrm{L}^{-1}\right)$ & 52,0 \\
\hline Nitrogênio Total $\left(\mathrm{mg} \mathrm{L}^{-1}\right)$ & 59,1 \\
\hline Fósforo Total $\left(\mathrm{mg} \mathrm{L}^{-1}\right)$ & 7,5 \\
\hline Sulfato $\left(\mathrm{mg} \mathrm{L}^{-1}\right)$ & 4,0 \\
\hline Sólidos Suspensos $\left(\mathrm{mg} \mathrm{L}^{-1}\right)$ & 57,0 \\
\hline $\begin{array}{l}\text { Sólidos Totais a } 105^{\circ} \mathrm{C}\left(\mathrm{mg} \mathrm{L}^{-1}\right) \\
\mathrm{pH}\end{array}$ & $\begin{array}{c}484,0 \\
7,0\end{array}$ \\
\hline Temperatura $\left({ }^{\circ} \mathrm{C}\right)$ & 22,0 \\
\hline Amoxicilina $\left(\mu \mathrm{g} \mathrm{L}^{-1}\right)$ & 27,0 \\
\hline Ciprofloxacin $\left(\mu \mathrm{g} \mathrm{L}^{-1}\right)$ & 54,0 \\
\hline Sulfametoxazol $\left(\mu \mathrm{g} \mathrm{L}^{-1}\right)$ & 27,7 \\
\hline Trimetroprima $\left(\mu \mathrm{g} \mathrm{L}^{-1}\right)$ & 6,6 \\
\hline
\end{tabular}

Neste contexto nota-se a necessidade de um tratamento eficaz para este efluente assim utilizou-se um reator do tipo RBC (Rotating Biological Contact), que foi instalado primeiramente na década de 1960 na Alemanha e vem sendo utilizado por diversas empresas para o tratamento de seus efluentes atualmente.

O RBC consiste em uma série de discos circulares como material suporte para o crescimento de microrganismos com espaçamento bem próximo, onde os mesmos são submersos no efluente e girados vagarosamente (METCALF \& EDDY, 1991). Este reator constitui-se como uma tecnologia comprovadamente eficiente no tratamento de efluentes oferecendo vantagens como: ambiente com baixa tensão de cisalhamento, fácil scale up, alta área de superfície por unidade de volume, baixos custos de manutenção, baixo requerimento de energia e simples construção e operação (GUIMARÃES et al., 2005). 


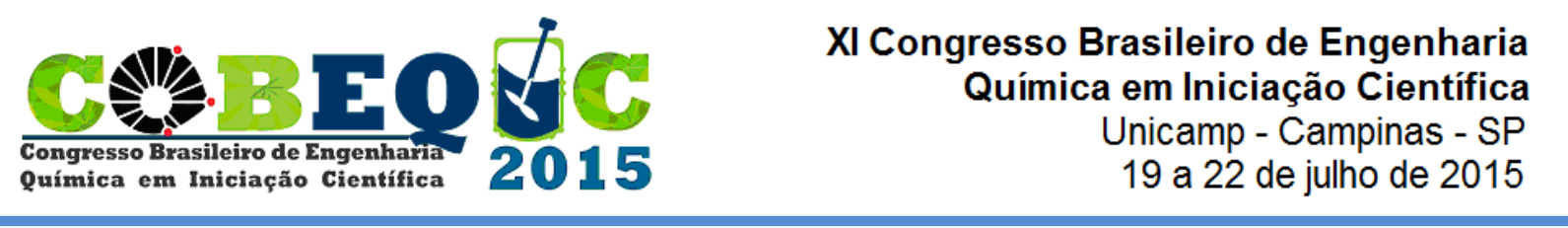

\section{OBJETIVO}

O presente trabalho tem como objetivo avaliar a eficiência de um reator do tipo RBC em escala de bancada na remoção de carga orgânica de despejos líquidos provenientes do Hospital Universitário de Santa Maria.

\section{METODOLOGIA}

No estudo realizado as amostras foram coletadas na parte sul do hospital universitário, após a fossa séptica e o filtro anaeróbio, as mesmas eram armazenadas em toneis de 50 litros e conduzidas ao laboratório onde ficava o sistema para o estudo.

O reator utilizado possui formato tubular e é constituído de um tubo de PVC com as seguintes medidas: diâmetro interno de $13 \mathrm{~cm}$ e comprimento de $94 \mathrm{~cm}$, volume útil de 5 litros e o suporte para o biofilme sendo CD's que possuem diâmetro de $12 \mathrm{~cm}, 1 \mathrm{~mm}$ de espessura, raio interno de $0,75 \mathrm{~cm}$ e raio externo de $6 \mathrm{~cm}$. Na figura 1 pode-se observar o fluxograma do processo. O reservatório, de cor azul claro, corresponde à alimentação do reator e o reservatório, azul escuro, corresponde ao efluente tratado.

Figura 1 - fluxograma do funcionamento do RBC

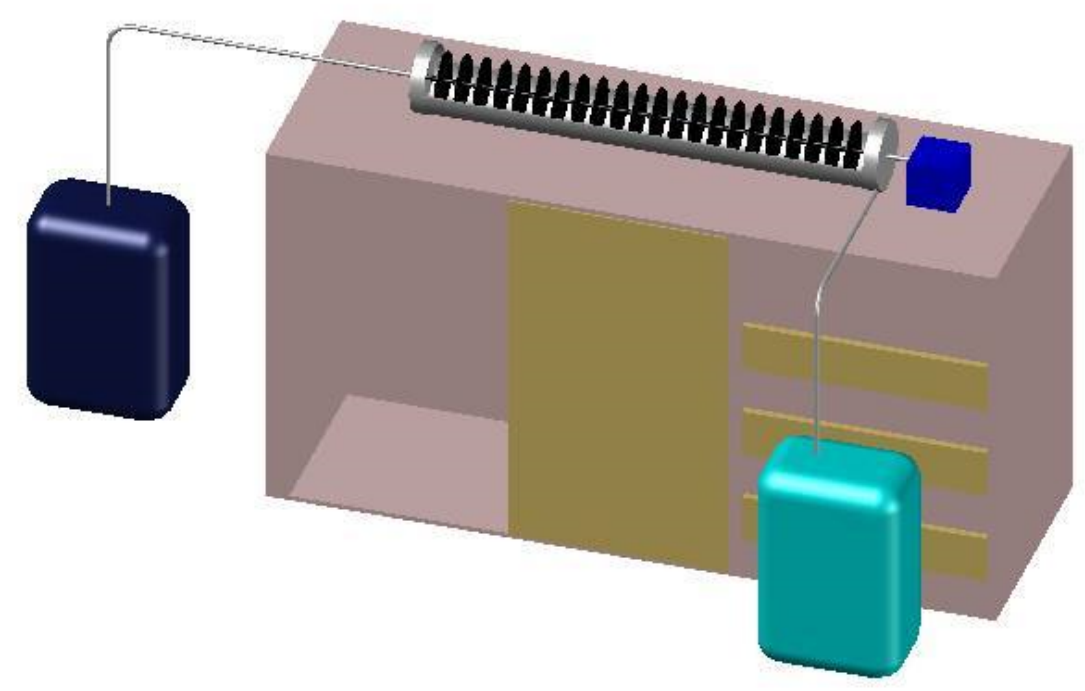

Coletava-se o efluente de estudo três vezes por semana, armazenando-o em um recipiente de 50 litros do qual era transferido para o reator por meio de uma bomba peristáltica (Provitec-Série DM 500), com uma vazão de $1 \mathrm{~L} / \mathrm{h}$ e com Tempo de Retenção Hidráulico para o efluente de $5 \mathrm{~h}$. O eixo move-se por um motor de baixa potencia e pequeno consumo de energia, com velocidade de rotação de 3 RPM. 
Para a partida do reator, inicialmente utilizou-se lodo gerado por outro experimento de tratamento biológico realizado no laboratório que estava em andamento há mais tempo.

Após a entrada do reator em processo contínuo, iniciaram-se as análises, que tiveram como base o standard methods. O teste de DQO, utilizado para medir a quantidade de oxigênio dissolvido em meio ácido para degradar a matéria orgânica e também estimar a demanda oxidante residual de matéria orgânica no esgoto, teve como objeto de oxidação o dicromato de potássio, um forte oxidante químico.

Em muitos casos, o resultado da DQO dá uma boa estimativa de valor para a DBO, quando relacionadas. A estabilização completa, nas análises de DBO, demora, na prática, vários dias. Todavia, após 5 dias 67 a $75 \%$ da DBO é satisfeita para a maioria dos esgotos domésticos (MACEDO, J. A., 2006). Por isso neste estudo utilizou-se um período de tempo de incubação de cinco dias, onde obtemos a $\mathrm{DBO}_{5}$.

\section{RESUTADOS E DISCUSSÃO}

Os resultados aqui apresentados fazem parte de uma analise preliminar de resultados englobando apenas os 40 primeiros dias de operação continua do reator RBC.

Durante os 40 primeiros dias de operação foram realizadas 6 análises em que foram determinadas as concentrações de $\mathrm{DQO}$ e $\mathrm{DBO}_{5}$ do efluente e do afluente. Os resultados obtidos são representados na figura 2.

Figura 2- Eficiência de oxidação de DQO e DBO5

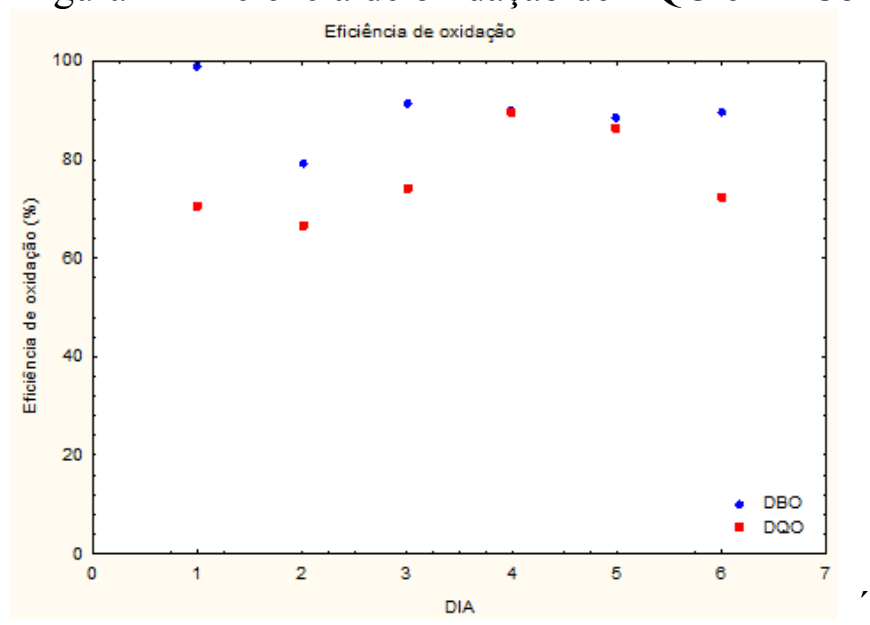

Os parâmetros analisados, conforme o Standard Methods for Examination of Water and Wastewater, com resultados obtidos para DQO de $520 \mathrm{mg} / \mathrm{L}$ para a entra e $120 \mathrm{mg} / \mathrm{L}$ para a saída, e para a $\mathrm{DBO}_{5}$ uma entrada de $250 \mathrm{mg} / \mathrm{L}$ e saída de $26 \mathrm{mg} / \mathrm{L}$.

Calcularam-se as remoções individuais, que podem ser vistas no gráfico da figura 4, e a partir das concentrações de entrada e saída calculou-se as eficiências médias de remoção, 
sendo obtida $77 \%$ para a demanda química de oxigênio e de $89,6 \%$ para a demanda bioquímica de oxigênio ao quinto dia.

\section{CONCLUSÃO}

A Resolução da CONAMA No 430/2011 onde "Dispões sobre condições e padrões de lançamento de efluentes, complementa e altera a Resolução n ${ }^{\circ}$ 357, de 17 de março de 2005", capítulo II, seção III- Das Condições e Padrões para Efluentes de Sistemas de Tratamento de Esgotos Sanitários, dispõem o limite de máximo da Demanda Bioquímica de Oxigênio com 5 dias de digestão, é de $120 \mathrm{mg} / \mathrm{L}$, sendo que este limite pode ser ultrapassado no caso em que o tratamento alcançar eficiência maiores que $60 \%$.

Assim os resultados obtidos foram satisfatórios, enquadrando-se dentro da legislação ambiental segundo a resolução citada acima. Com os valores obtidos para a DQO e $\mathrm{DBO}_{5}$, conclui-se que os Reatores de Biológicos de Contato são, em principio, uma boa opção para o tratamento de efluentes hospitalares ainda que sejam resultados preliminares. 


\section{REFERÊNCIAS}

MACEDO, J. A., Introdução à química ambiental, Juiz de Fora, MG, CRQ-MG, 2006, 2a Ed., $1028 \mathrm{p}$.

KÜMMERER, Klaus. Pharmaceuticals in the Environment. $2^{\text {nd }}$ ed. Alemanha: Springer, 2004.

MINETTO, L. Reatores de discos rotativos e tubular helicoidal na degradação fotocatalítica de diclofenaco e carga orgânica de efluente hospitalar. RS. 2009. Dissertação (Mestrado em Química) - Universidade Federal de Santa Maria, Santa Maria, RS, Brazil, 2009.

METCALF \& EDDY, 1991, Wastewater Engineering - Treatment, Disposal and Reuse. 3rd edition, McGraw-Hill, USA.

APHA/AWWA/WEF. Standard Methods for the Examination of Water and Wastewater. 18. ed. Washington: American Public Health Association, 1998.

GUIMARÃES, C.; PORTO, P.; OLIVEIRA, R.; MOTA, M. (2005). Continuous decolourization of a sugar refinery wastewater in a modified rotating biological contactor with Phanerochaete chrysosporium immobilized on polyurethane foam disks. Process Biochemistry, n. 40, p.535-540 